\title{
Assessment of sustainable and biodegradable agricultural substrates for eminence production of cucumber for kitchen gardening
}

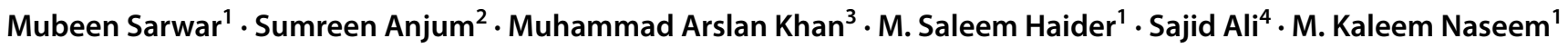

Received: 11 September 2017 / Accepted: 24 September 2018 / Published online: 1 October 2018

(c) The Author(s) 2018

\begin{abstract}
Purpose Different agricultural substrates were evaluated for growth and productivity of cucumber under pot culture for kitchen gardening.

Methods Several agricultural substrates such as leaf compost, compost, perlite, and coconut compost were used in with silt in several combinations. Seeds were sown in plastic pots of 9-L capacity and filled with growing substrates in various combinations. The study consisted of eight treatments; each treatment had four replicates.

Results Use of different growing media alone or in combination significantly enhanced plant growth and productivity, compared to control. Results showed that maximum germination, plant growth, emergence percentage, gas exchange attributes, shoot/root length, shoot fresh and dry biomass, root fresh and dry biomass, no. of leaves, leaf mineral contents, and chlorophyll concentration and yield was noted in the plants grown in leaf compost + perlite + silt $(1: 1: 1)$ media combination. In case of proline contents, all treatment combinations showed non-significant; but, NPK status varied because of the presence of organic matter in substrates that ultimately enhanced the nutrient uptake of cucumber plants.

Conclusions Although all media either alone or in combination positively influenced different studied parameters of cucumber, but, leaf compost + perlite + silt $(1: 1: 1)$ combination was the best. Therefore, leaf compost + perlite + silt $(1: 1: 1)$ media combination could be considered suitable for the cucumber cultivation in the form of kitchen gardening.
\end{abstract}

Keywords Agricultural substrates $\cdot$ Potting medium $\cdot$ Perlite $\cdot$ Gas exchange $\cdot$ Proline

\section{Introduction}

Vegetables are good source of various phyto-chemicals and nutrients, which are considered vital for several metabolic processes in the human body (Noreen and Ashraf 2009). Cucumber (Cucumis sativus L.) is an important vegetable crop (Stepien and Klobus 2006). It belongs to the family Cucurbitaceae, native to Africa and Asia where it has

Mubeen Sarwar

mubeensarwar4@yahoo.com

1 Institute of Agricultural Sciences, University of the Punjab, Lahore 54590, Pakistan

2 Department of Botany, University of Agriculture, Faisalabad 38000, Pakistan

3 Department of Plant Pathology, Mian Nawaz Sharif University of Agriculture, Multan, Pakistan

4 Department of Horticulture, Faculty of Agricultural Sciences and Technology, Bahauddin Zakariya University, Multan 60800, Pakistan been used for 3000 years. Cucumbers normally picked at mature green stage and are eaten as a fresh vegetable. It is also a rich source of niacin, vitamin $\mathrm{C}$, thiamine, calcium, iron, phosphorus, and fiber (Gopalan et al. 1989). Increasing urbanization and food security are among the key issues of the present era (FAO 2011). Billions of the people around the world are unable to purchase or have the access to sufficient food for themselves and their families (Nkosi et al. 2014) which causes the malnutrition and leads to poor health. It also retards physical and mental growth or ultimately enhances the risk of illness and decreases work output. For poor people, fruits and vegetables are the only source of nutrients. Kitchen/home gardening is one of the oldest food production practices around the world (Landauer 1985). Production of vegetables and fruits at household level provides direct access to essential nutrients which may not be available for poor people. Therefore, in this regard, home/ kitchen gardening could be a good source to combat with food security (Talukder et al. 2002a, b). Problems of soils such as soil borne pathogen, shortage of water for irrigation, 
and lack of suitable soils are urging for using of soilless culture for vegetable production (Dorais et al. 2001). Despite various soilless cultures, substrate (aggregate) culture is gaining popularity around the world (Gul et al. 2005). Several features are considered critical for selecting materials for soilless pot growth media such as high water holding capacity, good draining, high ionic exchange capacity, and their devoid of pests, pathogens, and weeds (Cantliffe et al. 2003). Therefore, cultivation of plants in pots is basically influenced by a lot of soilless cultures (Wilson et al. 2003; Younis et al. 2011) and most vital cultural contribution is growing substrate which is pre-requisite for the growth and development of plants (Younis et al. 2013). Media for plant growth and constituents of potting soil have gained attraction for a number of decades (Maloupa et al. 1992). The best medium gives adequate anchorage and supports plant body and allows nutrients for diffusion of oxygen towards the rhizosphere (roots) and permits gaseous exchange among the rhizosphere and atmosphere. However, each growing substrate has many types of structure and materials which might have a direct or indirect impact on the plant growth. The use of various media either alone in combination is considered highly productive (Nair et al. 2011; Vaughn et al. 2011). Therefore, selection of good substrate is vital for good plant productivity (Olympios 1999). A perfect growing media combination which is used for the cultivation of greenhouse crops should have four basic properties, provide a suitable anchorage for maximum root growth, deliver the proper aeration and water, produce a reservoir of essential nutrient uptake, and ensure physical support to the plants (Tariq et al. 2012). Some inorganic/mineral potting substrates are being used for commercial and domestic purposes. A number of studies reported that it is very easy to tackle soilless media cultivation (Yasmeen et al. 2012). Some findings have suggested that peat can be replaced through artificial fertilizers deprived of any negative effect on plant growth and productivity (Larcher and Scariot 2009). It has also been reported that growing media significantly affect the morphology and productivity of plants (Savvas et al. 2004; Ghazvini et al. 2007). The varied proportion of agricultural substrate has a thoughtful impact on biological, chemical, and physical properties of the media with altered microbial activity (Alidoust et al. 2012), which eventually reduces the loss of nitrogen and enhances the cation exchange capacity (CEC). Such type of compost or mixtures of substrates performs as a good conditioner of the soil (Bulluck et al. 2002; Arancon et al. 2004). Good potting media perform several functions such as increase in various aspects of plant such as fresh and dry weights (Wang and Lin 2006), number of leaves (Eklind et al. 2001), chlorophyll contents (Hashemimajd et al. 2004), and improved mineral nutrient availability (Zaller 2007), though specific scientific information about cucumber pot production for kitchen gardening is insufficient in Pakistan.
Therefore, keeping in view the significance of cucumber and the role of the agricultural growing media in its growth, the present research was conducted to study the effectiveness of various growing substrates (perlite, compost, silt, peat moss, and leaf compost) either alone or in combination for the growth and productivity of the cucumber.

\section{Materials and methods}

An experiment was conducted at the Institute of Horticultural Sciences, University of Agriculture, Faisalabad, Pakistan, for the evaluation of five different growing media either alone or in combination on the volume basis with each other, viz., leaf manure, compost (prepared from farm yard manure), peat moss, perlite, and coconut peat for cucumber cv. 'Green Long' in pot production. Seeds were sown in plastic pots having 9-L capacity and study was comprised of eight treatments and each treatment had four replicates. Treatments were $\mathrm{T}_{1}=\operatorname{silt}($ Control $) ; \mathrm{T}_{2}=$ compost $+\operatorname{silt}(1: 1)$; $\mathrm{T}_{3}=$ leaf compost + silt $(1: 1) ; \mathrm{T}_{4}=$ coconut peat + silt $(1: 1)$; $\mathrm{T}_{5}=$ perlite + silt $(1: 1) ; \mathrm{T}_{6}=$ compost + leaf compost + silt $(1: 1: 1) ; \mathrm{T}_{7}=$ coconut peat + perlite + silt $;(1: 1: 1) ; \mathrm{T}_{8}=$ coconut peat + compost + leaf compost + perlite + silt (1:1:1:1:1). Five grams of soluble NPK fertilizer were added into each pot once and cultural practices, irrigation, fertilizer application, and weeding, and pesticide application was same for the whole growth period, and pots were kept under greenhouse $\left(30-35{ }^{\circ} \mathrm{C}\right.$ and relative humidity $\left.40-50 \%\right)$. All pots were irrigated after 2 day intervals.

\section{Preparation of media and analysis}

For compost preparation, garden residues such as leaf clippings and pruning were used after 100 days of decomposition process kept under $\left(62{ }^{\circ} \mathrm{C}\right)$ in the bin. Chemical analysis of media was done before the start of the study, and all media recipes were subjected to chemical examination such as $\mathrm{pH}, \mathrm{EC}$, organic matter $(\mathrm{OM})$, nitrogen $(\mathrm{N})$, phosphorus $(\mathrm{P})$, and potassium $(\mathrm{K})$ determination as mentioned in Table 1. The $\mathrm{pH}$ was determined with the help of digital ion analyzer (Thomas 1996). The percentage of $\mathrm{N}$ in the substrates was assessed by distillation in "Kjeldahl's apparatus" and titration was carried out with the standard $\mathrm{H}_{2} \mathrm{SO}_{4}$, whereas methyl red and boric acid were used as indicators (Jackson 1962; Bremner and Mulvaney 1982). For the estimation of $\mathrm{P}$ in media, Olsen's method was used (Watanabe and Olsen 1965; Olsen et al. 1984), and for the estimation of K, ion flame photometer method was used (U.S. Salinity Lab. Staff 1954). 
Table 1 Chemical characteristics of growing media

\begin{tabular}{|c|c|c|c|c|c|c|c|}
\hline & Treatments & $\mathrm{pH}$ & $\mathrm{EC}\left(\mathrm{dS} \mathrm{m} \mathrm{m}^{-1}\right)$ & $\mathrm{OM}(\%)$ & $\mathrm{N}(\%)$ & $\mathrm{P}\left(\mathrm{mg} \mathrm{L}^{-1}\right)$ & $\mathrm{K}\left(\mathrm{mg} \mathrm{L}^{-1}\right)$ \\
\hline $\mathrm{T}_{1}$ & Silt (control) & 6.70 & 1.23 & 0.53 & 0.28 & 3.05 & 110 \\
\hline $\mathrm{T}_{2}$ & Compost + silt & 6.90 & 2.3 & 0.81 & 1.34 & 2.47 & 160 \\
\hline $\mathrm{T}_{3}$ & Leaf compost + silt & 6.98 & 1.98 & 1.22 & 1.64 & 10.14 & 218 \\
\hline $\mathrm{T}_{4}$ & Coconut peat + silt & 6.60 & 3.12 & 1.38 & 1.98 & 17.23 & 270 \\
\hline $\mathrm{T}_{5}$ & Perlite + silt & 6.50 & 2.01 & 2.10 & 1.60 & 12.32 & 190 \\
\hline $\mathrm{T}_{6}$ & Compost + leaf compost + silt & 6.30 & 2.15 & 1.10 & 1.89 & 20.41 & 360 \\
\hline $\mathrm{T}_{7}$ & Coconut peat + perlite + silt & 6.20 & 2.16 & 1.82 & 2.10 & 14.58 & 380 \\
\hline $\mathrm{T}_{8}$ & $\begin{array}{l}\text { Coconut peat }+ \text { compost }+ \text { leaf } \\
\text { Compost }+ \text { perlite }+ \text { silt }\end{array}$ & 6.60 & 2.12 & 2.12 & 2.31 & 22.60 & 430 \\
\hline
\end{tabular}

$\mathrm{T}_{1}=$ silt (control); $\mathrm{T}_{2}=$ compost + silt $(1: 1) ; \mathrm{T}_{3}=$ leaf compost + silt $(1: 1) ; \mathrm{T}_{4}=$ coconut peat + silt $(1: 1)$; $\mathrm{T}_{5}=$ perlite + silt $(1: 1) ; \mathrm{T}_{6}=$ compost + leaf compost + silt $(1: 1: 1) ; \mathrm{T}_{7}=$ coconut peat + perlite + silt $;(1: 1: 1)$ and $\mathrm{T}_{8}=$ coconut peat + compost + leaf compost + perlite + silt $(1: 1: 1: 1: 1)$

\section{Mean emergence time (MET)}

Daily visited the experiment and noted the time when the first seed started to emerge. Mean emergence time (MET) in days was recorded by the method of Ellis and Roberts (1981) as mentioned below:

$\mathrm{MET}=\sum \mathrm{Dn}$

$$
\sum \text { n }
$$

where $N$ number of seeds emerge on day $D$, and $D$ number of days counted from beginning of the test.

\section{Emergence index (EI)}

Emergence index (EI) was calculated as defined method in the handbook of the AOSA (1990) as follows:

$\mathrm{EI}=\quad$ Number of emerged seeds

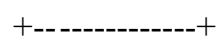

Day of first count

\section{Final emergence percentage (FEP) \%}

Final cucumber seed emergence percentage (\%) was calculated at the end of the investigation by taking number of seeds emerged to total seeds sown.

\section{Morphological traits}

Following parameters were noted including shoot and root length $(\mathrm{cm})$ with measuring scale, number of leaves plant ${ }^{-1}$, shoot fresh and dry biomass (g), and root fresh and dry biomass $(\mathrm{g})$ were calculated using weight balance (Sarwar et al. 2013, 2017).

\section{Chlorophyll contents (SPAD)}

Chlorophyll contents (SPAD) were measured by chlorophyll meter (Model: SPAD: 502, Minolta; Japan) with the stated procedure as reported by Sarwar et al. (2017).

\section{Measurement of proline contents $\left(\mu \mathrm{mol} \mathrm{g} \mathrm{g}^{-1} \mathrm{FW}\right.$ )}

The proline was calculated by the procedure of Bates et al. (1972) and expressed as $\mu \mathrm{mol} \mathrm{g}^{-1} \mathrm{FW}$ :

Mole proline $\mathrm{g}^{-1}$ fresh weight

$=\left[\mathrm{g}\right.$ proline $\mathrm{ml}^{-1} \times \mathrm{ml}$ of toluene $\left./ 115.5\right) /$

(g of sample/5)].

Number of emerged seeds

Days of final count

\section{Gas exchange attributes}

Gas exchange traits' measurement was noted from the top third leaf of each cucumber plant. The readings were taken between 11:00 am and 1:30 pm with the specified methods of Zekri (1991) and Moya et al. (2003).

\section{Yield attributes}

\section{Number of fruits plant ${ }^{-1}$}

The cucumber plants from each replication were randomly selected and the number of fruit plant ${ }^{-1}$ was recorded, 
and the average of each replication in each treatment was computed.

\section{Fruit average weight (g)}

Four cucumber plants from each replication were randomly selected and average fruit weight (g) was weighed using a digital balance.

\section{Total cucumber yield per plant $(g)$}

After harvesting of cucumber from each plant, weigh the weight of fruit and note separately as cucumber weight plant $^{-1}$, and the average of each replication in each treatment was computed.

\section{Leaf nutrient estimation}

Chapman and Parker's (1961) method was used for the estimation of $\mathrm{N}$ contents (\%), $\mathrm{P}(\mathrm{mg} / \mathrm{L} \mathrm{DW})$, and potassium (mg/L DW) in dry cucumber leaves. P was estimated by spectrophotometer (Model; U: 2020) and $\mathrm{K}^{+}$ions were calculated with the procedure of Chapman and Parker (1961) using flame photometer (Flame Photometer: 410).

\section{Statistical analysis}

Study was carried out under completely randomized design and data were analyzed through analysis of variance technique to find out the differences among the treatment means. The least significance difference (LSD) test was applied at a significance level of 5\% with Statistix 8.1 software (Steel et al. 1997).

\section{Results}

\section{Morphological attributes of cucumber}

\section{Mean emergence time (MET) days}

Different growing media markedly influenced different morphological attributes of cucumber (Table 2). The lowest mean emergence time was noted in $\mathrm{T}_{8}$ (coconut peat + compost + leaf compost + perlite + silt) as this growing medium revealed earliest germination of cucumber seeds by taking only 5.05 days, as compared to all other treatments (Table 2).

\section{Emergence index (EI)}

The used growing media had a significant effect on emergence index of cucumber plants (Table 2). Results exhibited that maximum emergence index (10.87) was noted in $\mathrm{T}_{8}$ (coconut peat + compost + leaf compost + perlite + silt $)$, compared to all other treatments (Table 2). In contrast, lowest emergence index (6.88) was noted in $\mathrm{T}_{1}$ (silt).

\section{Final emergence percentage \% (FEP)}

Different growing media had a significant effect on final emergence percentage (Table 2). Among other treatments, the minimum emergence index (6.88) was noticed in $\mathrm{T}_{1}$ (silt). The results for final emergence percentage showed that $\mathrm{T}_{8}$ (coconut peat + compost + leaf compost + perlite + silt $)$ resulted in the maximum emergence percentage $(91.25 \%)$, as compared to other treatments (Table 2). Among other treatments, a non-significant difference was observed in $\mathrm{T}_{4}$ (78.75\%) and $\mathrm{T}_{3}(80.0 \%)$.

Table 2 Effect of different growing media on germination and morphological attributes of cucumber

\begin{tabular}{lllllll}
\hline Treatments & MET & EI & FEP $(\%)$ & SL $(\mathrm{cm})$ & RL $(\mathrm{cm})$ & SFW $(\mathrm{g})$ \\
\hline $\mathrm{T}_{1}$ & $8.56 \pm 0.05 \mathrm{a}$ & $6.88 \pm 0.18 \mathrm{~d}$ & $81.25 \pm 2.39 \mathrm{~b}$ & $43.60 \pm 2.09 \mathrm{de}$ & $24.65 \pm 0.65 \mathrm{e}$ & $16.34 \pm 0.75 \mathrm{e}$ \\
$\mathrm{T}_{2}$ & $8.28 \pm 0.05 \mathrm{~b}$ & $8.47 \pm 0.10 \mathrm{c}$ & $82.50 \pm 2.50 \mathrm{~b}$ & $50.64 \pm 3.60 \mathrm{bcd}$ & $30.54 \pm 2.33 \mathrm{~cd}$ & $19.60 \pm 0.73 \mathrm{de}$ \\
$\mathrm{T}_{3}$ & $8.22 \pm 0.05 \mathrm{bc}$ & $8.54 \pm 0.22 \mathrm{c}$ & $80.00 \pm 2.04 \mathrm{~b}$ & $44.63 \pm 2.66 \mathrm{cde}$ & $26.52 \pm 2.02 \mathrm{de}$ & $18.34 \pm 0.69 \mathrm{de}$ \\
$\mathrm{T}_{4}$ & $8.26 \pm 0.05 \mathrm{~b}$ & $8.29 \pm 0.23 \mathrm{c}$ & $78.75 \pm 2.39 \mathrm{~b}$ & $52.80 \pm 3.65 \mathrm{bc}$ & $30.26 \pm 1.34 \mathrm{cde}$ & $20.78 \pm 1.94 \mathrm{de}$ \\
$\mathrm{T}_{5}$ & $8.24 \pm 0.03 \mathrm{bc}$ & $9.17 \pm 0.41 \mathrm{bc}$ & $81.25 \pm 3.15 \mathrm{~b}$ & $47.35 \pm 4.98 \mathrm{bcde}$ & $33.56 \pm 2.70 \mathrm{abc}$ & $22.30 \pm 1.83 \mathrm{~cd}$ \\
$\mathrm{~T}_{6}$ & $8.24 \pm 0.07 \mathrm{bc}$ & $9.71 \pm 0.51 \mathrm{~b}$ & $83.75 \pm 1.25 \mathrm{~b}$ & $41.17 \pm 2.01 \mathrm{e}$ & $31.12 \pm 1.75 \mathrm{bcd}$ & $25.55 \pm 2.87 \mathrm{bc}$ \\
$\mathrm{T}_{7}$ & $8.21 \pm 0.08 \mathrm{bc}$ & $9.01 \pm 0.46 \mathrm{bc}$ & $81.50 \pm 0.96 \mathrm{~b}$ & $55.99 \pm 0.91 \mathrm{ab}$ & $37.43 \pm 1.84 \mathrm{a}$ & $27.08 \pm 3.12 \mathrm{ab}$ \\
$\mathrm{T}_{8}$ & $8.05 \pm 0.08 \mathrm{c}$ & $10.87 \pm 0.42 \mathrm{a}$ & $91.25 \pm 2.39 \mathrm{a}$ & $62.72 \pm 3.09 \mathrm{a}$ & $36.62 \pm 2.38 \mathrm{ab}$ & $30.36 \pm 1.35 \mathrm{a}$ \\
\hline
\end{tabular}

$\mathrm{T}_{1}=$ silt $($ control $) ; \mathrm{T}_{2}=$ compost $+\operatorname{silt}(1: 1) ; \mathrm{T}_{3}=$ leaf compost + silt $(1: 1) ; \mathrm{T}_{4}=$ coconut peat + silt $(1: 1) ; \mathrm{T}_{5}=$ perlite + silt $(1: 1) ; \mathrm{T}_{6}=$ compost + leaf compost + silt $(1: 1: 1) ; \mathrm{T}_{7}=$ coconut peat + perlite + silt; $(1: 1: 1)$ and $\mathrm{T}_{8}=$ coconut peat + compost + leaf compost + perlite + silt $(1: 1: 1: 1: 1)$

$M E T$ mean emergence time, $E I$ emergence index, FEP final emergence percentage, $S L$ shoot length, $R L$ root length, $S F W$ shoot fresh weight 


\section{Shoot and root length $(\mathrm{cm})$}

Different growing media showed significant differences in shoot and root length (Table 2). The results showed that highest shot length was recorded in $\mathrm{T}_{8}$ (coconut peat + compost + leaf compost + perlite + silt). The shoot length of $T_{8}$ (coconut peat + compost + leaf compost + perlite + silt $)$ was $62.72 \mathrm{~cm}$, as compared to $\mathrm{T}_{6}$ (compost + leaf compost + silt) which showed minimum length of shoot $(41.17 \mathrm{~cm})$. As far as root length is concerned, it was observed maximum $(37.43 \mathrm{~cm})$ in $\mathrm{T}_{7}$ (coconut peat + perlite + silt), as compared to $\mathrm{T}_{1}$ (silt) which had minimum root length $(24.65 \mathrm{~cm})$.

\section{Shoot and root fresh weight $(g)$}

Shoot and root fresh weight was also substantially influenced in response to different used growing media for cucumber (Table 2,3). Results exhibited highest shoot fresh weight $\left(30.36 \mathrm{~g}\right.$ ) was observed in $\mathrm{T}_{8}$ (coconut peat + compost + leaf compost + perlite + silt), in comparison to $\mathrm{T}_{1}$ (silt) which had minimum (16.43 g) shoot fresh weight (Table 2). As far as root fresh weight is concerned, it was found to be the highest ( $3.91 \mathrm{~g}$ ) in $\mathrm{T}_{8}$ (coconut peat + compost + leaf compost + perlite + silt). On the other side, lowest root fresh weight $(1.96 \mathrm{~g})$ was noted in treatment $\mathrm{T}_{5}$ (perlite + silt) Table 3.

\section{Shoot and root dry weight (g)}

Different growing media had a significant influence on shoot and root dry weight (Table 3). Results revealed that maximum shoot dry weight $\left(7.29 \mathrm{~g}\right.$ ) was noted in $\mathrm{T}_{8}$ (coconut peat + compost + leaf compost + perlite + silt), in contrast to $\mathrm{T}_{1}$ (silt) that resulted in lowest shoot dry weight (2.43 g). As far as root dry weight is concerned, it was observed that $\mathrm{T}_{8}$ (coconut peat + compost + leaf compost + perlite + silt) produced maximum root dry weight (3.91 g), compared
$\mathrm{T}_{5}$ in which lowest root dry weight $(1.96 \mathrm{~g})$ was obtained (Table 3).

\section{Number of leaves}

Different treatments showed different response to number of leaves (Table 3 ). It was noted that $\mathrm{T}_{8}$ (coconut peat + compost + leaf compost + perlite + silt) exhibited maximum number of leaves (18.89), as compared to $\mathrm{T}_{1}$ (silt) which resulted in minimum number of leaves (13.87). In contrast, rest all treatments showed non-significant differences for number leaves of cucumber plants (Table 3).

\section{Chlorophyll contents (SPAD)}

Substantial differences in chlorophyll contents of cucumber leaves were noted in response various used growing media (Table 3). Mixture of all media coconut peat + compost + leaf compost + perlite + silt $\left(\mathrm{T}_{8}\right)$ in the same ratio significantly enhanced the chlorophyll contents (41.14 SPAD) in the cucumber leaves, compared to different other treatments. On the other hand, treatments $\mathrm{T}_{2}$ (compost + silt coconut peat + silt $), \mathrm{T}_{3}$ (leaf compost + silt), $\mathrm{T}_{4}$ (coconut peat + silt), and $\mathrm{T}_{6}$ (compost + leaf compost + silt) were statistically at par with each other having 28.73, 29.83, 27.70, and 29.42 SPAD values, respectively. Overall, T5 showed minimum leaf chlorophyll contents (24.56 SPAD) among all treatments (Table 3).

\section{Proline contents ( $\left.\mu \mathrm{mol} \mathrm{g}^{-1} \mathrm{FW}\right)$}

Different growing media markedly affected proline contents of cucumber leaves (Table 3). Results showed that combination of coconut peat + silt $\left(\mathrm{T}_{4}\right)$ had higher proline contents $\left(13.75 \mu \mathrm{mol} \mathrm{g}^{-1} \mathrm{FW}\right)$ than various other treatments. On the other hand, $\mathrm{T}_{5}$ (perlite + silt) treatment showed minimum

Table 3 Effect of different growing media on morpho-physiological and biochemical attributes of cucumber

\begin{tabular}{lllllll}
\hline Treatments & RFW $(\mathrm{g})$ & SDW $(\mathrm{g})$ & RDW $(\mathrm{g})$ & NOL & CC $(\mathrm{SPAD})$ & PC $\left(\mu \mathrm{mol} \mathrm{g}^{-1} \mathrm{FW}\right)$ \\
\hline $\mathrm{T}_{1}$ & $5.61 \pm 0.35 \mathrm{c}$ & $2.43 \pm 0.05 \mathrm{~d}$ & $1.22 \pm 0.09 \mathrm{c}$ & $13.87 \pm 0.89 \mathrm{~b}$ & $25.12 \pm 1.64 \mathrm{~cd}$ & $8.50 \pm 0.65 \mathrm{~b}$ \\
$\mathrm{~T}_{2}$ & $5.77 \pm 0.39 \mathrm{c}$ & $3.03 \pm 0.58 \mathrm{~d}$ & $1.49 \pm 0.12 \mathrm{c}$ & $15.73 \pm 0.08 \mathrm{ab}$ & $28.73 \pm 0.77 \mathrm{~cd}$ & $12.82 \pm 1.37 \mathrm{a}$ \\
$\mathrm{T}_{3}$ & $6.58 \pm 0.40 \mathrm{bc}$ & $2.83 \pm 0.24 \mathrm{~d}$ & $1.65 \pm 0.16 \mathrm{c}$ & $15.09 \pm 0.14 \mathrm{ab}$ & $29.84 \pm 0.91 \mathrm{c}$ & $13.50 \pm 0.96 \mathrm{a}$ \\
$\mathrm{T}_{4}$ & $5.65 \pm 0.64 \mathrm{c}$ & $3.88 \pm 0.48 \mathrm{~cd}$ & $1.26 \pm 0.33 \mathrm{c}$ & $14.95 \pm 0.25 \mathrm{ab}$ & $27.70 \pm 2.06 \mathrm{~cd}$ & $13.75 \pm 0.75 \mathrm{a}$ \\
$\mathrm{T}_{5}$ & $6.21 \pm 0.42 \mathrm{bc}$ & $4.69 \pm 0.40 \mathrm{bc}$ & $1.63 \pm 0.56 \mathrm{c}$ & $15.31 \pm 0.29 \mathrm{ab}$ & $24.56 \pm 2.08 \mathrm{~d}$ & $11.75 \pm 0.85 \mathrm{a}$ \\
$\mathrm{T}_{6}$ & $7.78 \pm 0.95 \mathrm{~b}$ & $5.24 \pm 0.50 \mathrm{bc}$ & $2.35 \pm 0.25 \mathrm{~b}$ & $16.17 \pm 0.26 \mathrm{ab}$ & $29.92 \pm 1.82 \mathrm{c}$ & $14.25 \pm 1.25 \mathrm{a}$ \\
$\mathrm{T}_{7}$ & $9.60 \pm 0.48 \mathrm{a}$ & $6.04 \pm 0.85 \mathrm{ab}$ & $2.94 \pm 0.23 \mathrm{a}$ & $17.53 \pm 0.23 \mathrm{ab}$ & $35.40 \pm 2.10 \mathrm{~b}$ & $12.00 \pm 1.47 \mathrm{a}$ \\
$\mathrm{T}_{8}$ & $10.16 \pm 0.67 \mathrm{a}$ & $7.29 \pm 0.61 \mathrm{a}$ & $2.81 \pm 0.34 \mathrm{ab}$ & $18.89 \pm 0.25 \mathrm{a}$ & $41.14 \pm 1.59 \mathrm{a}$ & $13.50 \pm 0.65 \mathrm{a}$ \\
\hline
\end{tabular}

$\mathrm{T}_{1}=$ silt $($ control $) ; \mathrm{T}_{2}=$ compost + silt $(1: 1) ; \mathrm{T}_{3}=$ leaf compost + silt $(1: 1) ; \mathrm{T}_{4}=$ coconut peat + silt $(1: 1) ; \mathrm{T}_{5}=$ perlite + silt $(1: 1) ; \mathrm{T}_{6}=$ compost + leaf compost + silt $(1: 1: 1) ; \mathrm{T}_{7}=$ coconut peat + perlite + silt; $(1: 1: 1)$ and $\mathrm{T}_{8}=$ coconut peat + compost + leaf compost + perlite + silt $(1: 1: 1: 1: 1)$

$R F W$ root fresh weight, $S D W$ shoot dry weight, $R D W$ root dry weight, $N O L$ no. of leaves, $C C$ chlorophyll contents, $P C$ proline contents 
proline contents $\left(11.75 \mu \mathrm{mol} \mathrm{g}^{-1} \mathrm{FW}\right)$ of cucumber leaves among all other treatments (Table 3 ).

\section{Gas exchange attributes}

Different growing media markedly affected gas exchange characteristics of cucumber plants (Table 4). Among different gas exchange attributes, stomatal conductance was found to be maximum (7.15) in $\mathrm{T}_{8}$ (coconut peat + compost + leaf compost + perlite + silt), compared to the other treatments. As far as photosynthesis is concerned, it was also found maximum (5.08) in $\mathrm{T}_{8}$ (coconut peat + compost + leaf compost + perlite + silt $)$, in contrast to $T_{5}$ which resulted in the lowest photosynthesis rate (3.01) of cucumber plants (Table 4). The response of transpiration rate was also substantial in response to various growing media for cucumber plants. The results indicated that $\mathrm{T}_{8}$ (coconut peat + compost + leaf compost + perlite + silt) exhibited highest transpiration rate (3.55), in contrast to $\mathrm{T}_{3}$ which had lowest (2.49) rate of transpiration (Table 4).

\section{No of fruits plant ${ }^{-1}$}

The results for the number of fruits plant ${ }^{-1}$ of cucumber in response to different growing treatments were also significant (Table 4). Results revealed that maximum number of fruits plant ${ }^{-1}$ (14.30) was noted in $\mathrm{T}_{8}$ (coconut peat + compost + leaf compost + perlite + silt $)$, whereas, $\mathrm{T}_{6}(11.26)$ and $\mathrm{T}_{7}(11.28)$ presented an almost similar number of fruits plant $^{-1}$. In contrast, minimum number of fruits plant ${ }^{-1}$ (7.49) was observed in $\mathrm{T}_{1}$ (silt) and $\mathrm{T}_{2}$ (compost + silt).

\section{Average fruit weight (g)}

Statistically significant variations in average fruit weight of cucumber were observed in response to all used growing media (Table 4). In general, highest average fruit weight $(183.50 \mathrm{~g})$ was noted in $\mathrm{T}_{8}$ (coconut peat + compost + leaf compost + perlite + silt), as compared to the other treatments. In contrast, minimum average fruit weight $(98.25 \mathrm{~g})$ was observed in $\mathrm{T}_{1}$ (silt) sown cucumber plants (Table 4). On the other side, $\mathrm{T}_{7}$ (coconut peat + perlite + silt), $\mathrm{T}_{6}(\mathrm{com}-$ post + leaf compost + silt), and $\mathrm{T}_{5}$ (perlite + silt) were almost in the same range of average fruit weight having 139.50, 141.75 , and $146.25 \mathrm{~g}$, respectively.

\section{Total cucumber yield per plant (g)}

Different growing media's presented significant variations in response to cucumber yield (Fig. 1). Maximum cucumber yield was $\left(2642.05 \mathrm{~g}\right.$ ) exhibited by $\mathrm{T}_{8}$ (coconut peat + compost + leaf compost + perlite + silt), as compared to other treatments, whereas minimum cucumber yield $(692.96 \mathrm{~g})$ was observed in $\mathrm{T}_{3}$ (leaf compost + silt) as presented in Fig. 1. On the other side, $T_{6}$ (compost + leaf compost + silt) $\mathrm{T}_{7}$ (coconut peat + perlite + silt), which showed almost same yield, and $\mathrm{T}_{5}$ (perlite + silt) and $\mathrm{T}_{4}$ (perlite + silt) were also in the same range of cucumber fruit yield per plant, respectively.

\section{Mineral elements}

\section{Nitrogen contents (\%)}

Cucumber leaves showed significant variations in nitrogen (N) contents in response to all growing substrates (Table 5). Results exhibited that $\mathrm{T}_{8}$ (coconut peat + compost + leaf compost + perlite + silt) showed maximum $\mathrm{N}$ contents $(2.16 \%)$ in the cucumber leaves, compared to other treatments. On the other hand, the cucumber plants grown in $\mathrm{T}_{1}$ (silt) showed lowest $\mathrm{N}$ contents (1.19\%).

Table 4 Effect of different growing media on physiological and yield attributes of cucumber

\begin{tabular}{lllllc}
\hline Treatments & $\mathrm{Gs}\left(\mu \mathrm{mol} \mathrm{m} \mathrm{s}^{-1}\right)$ & $\mathrm{Pn}\left(\mu \mathrm{mol} \mathrm{m} \mathrm{s}^{-2}\right)$ & $\mathrm{E}\left(\mathrm{mmol} \mathrm{H}_{2} \mathrm{O} \mathrm{m}^{-2} \mathrm{~s}^{-1}\right)$ & $\mathrm{NF}$ & $\mathrm{AFW}(\mathrm{g})$ \\
\hline $\mathrm{T}_{1}$ & $5.25 \pm 0.21 \mathrm{~d}$ & $3.57 \pm 0.03 \mathrm{bc}$ & $2.62 \pm 0.03 \mathrm{~d}$ & $7.14 \pm 0.31 \mathrm{~cd}$ & $98.25 \pm 4.77 \mathrm{e}$ \\
$\mathrm{T}_{2}$ & $5.80 \pm 0.36 \mathrm{~cd}$ & $3.94 \pm 0.29 \mathrm{bc}$ & $2.66 \pm 0.033 \mathrm{~d}$ & $7.16 \pm 0.64 \mathrm{~cd}$ & $117.58 \pm 1.53 \mathrm{de}$ \\
$\mathrm{T}_{3}$ & $5.17 \pm 0.31 \mathrm{~d}$ & $3.29 \pm 0.02 \mathrm{c}$ & $2.59 \pm 0.05 \mathrm{~d}$ & $5.68 \pm 0.41 \mathrm{~d}$ & $122.00 \pm 5.00 \mathrm{~cd}$ \\
$\mathrm{~T}_{4}$ & $6.06 \pm 0.14 \mathrm{bcd}$ & $3.65 \pm 0.28 \mathrm{bc}$ & $2.96 \pm 0.25 \mathrm{~cd}$ & $8.71 \pm 0.91 \mathrm{c}$ & $138.75 \pm 3.12 \mathrm{bcd}$ \\
$\mathrm{T}_{5}$ & $6.55 \pm 0.48 \mathrm{abc}$ & $3.01 \pm 0.02 \mathrm{c}$ & $2.73 \pm 0.98 \mathrm{~cd}$ & $8.48 \pm 0.63 \mathrm{c}$ & $146.25 \pm 6.49 \mathrm{~b}$ \\
$\mathrm{~T}_{6}$ & $5.75 \pm 0.28 \mathrm{~cd}$ & $4.12 \pm 0.75 \mathrm{abc}$ & $3.26 \pm 0.29 \mathrm{bc}$ & $11.26 \pm 0.65 \mathrm{~b}$ & $141.75 \pm 7.85 \mathrm{bc}$ \\
$\mathrm{T}_{7}$ & $6.95 \pm 0.29 \mathrm{ab}$ & $4.48 \pm 0.65 \mathrm{ab}$ & $4.03 \pm 0.25 \mathrm{a}$ & $11.28 \pm 1.14 \mathrm{~b}$ & $139.50 \pm 6.20 \mathrm{bcd}$ \\
$\mathrm{T}_{8}$ & $7.15 \pm 0.30 \mathrm{a}$ & $5.08 \pm 0.24 \mathrm{a}$ & $3.55 \pm 0.35 \mathrm{ab}$ & $14.30 \pm 1.01 \mathrm{a}$ & $183.50 \pm 7.22 \mathrm{a}$ \\
\hline
\end{tabular}

$\mathrm{T}_{1}=$ silt $($ control $) ; \mathrm{T}_{2}=$ compost + silt $(1: 1) ; \mathrm{T}_{3}=$ leaf compost + silt $(1: 1) ; \mathrm{T}_{4}=$ coconut peat + silt $(1: 1) ; \mathrm{T}_{5}=$ perlite + silt $(1: 1) ; \mathrm{T}_{6}=$ compost + leaf compost + silt $(1: 1: 1) ; \mathrm{T}_{7}=$ coconut peat + perlite + silt; $(1: 1: 1)$ and $\mathrm{T}_{8}=$ coconut peat + compost + leaf compost + perlite + silt $(1: 1: 1: 1: 1)$

$g s$ stomatal conductance, $p n$ photosynthesis rate, $E$ transpiration rate, $N F$ no. of fruits/plants, $A F W$ average fruit weight 
Fig. 1 Effect of different growing media on total yield per plant of cucumber. $\mathrm{T}_{1}=$ silt (control); $\mathrm{T}_{2}=$ compost + silt $(1: 1) ; \mathrm{T}_{3}=$ leaf compost + silt $(1: 1) ; \mathrm{T}_{4}=$ coconut peat + silt $(1: 1) ; \mathrm{T}_{5}=$ perlite + silt $(1: 1)$; $\mathrm{T}_{6}=$ compost + leaf compost + silt $(1: 1: 1) ; \mathrm{T}_{7}=$ coconut peat + perlite + silt; $(1: 1: 1)$ and $\mathrm{T}_{8}$ $=$ coconut peat + compost + leaf compost + perlite + silt $(1: 1: 1: 1: 1)$

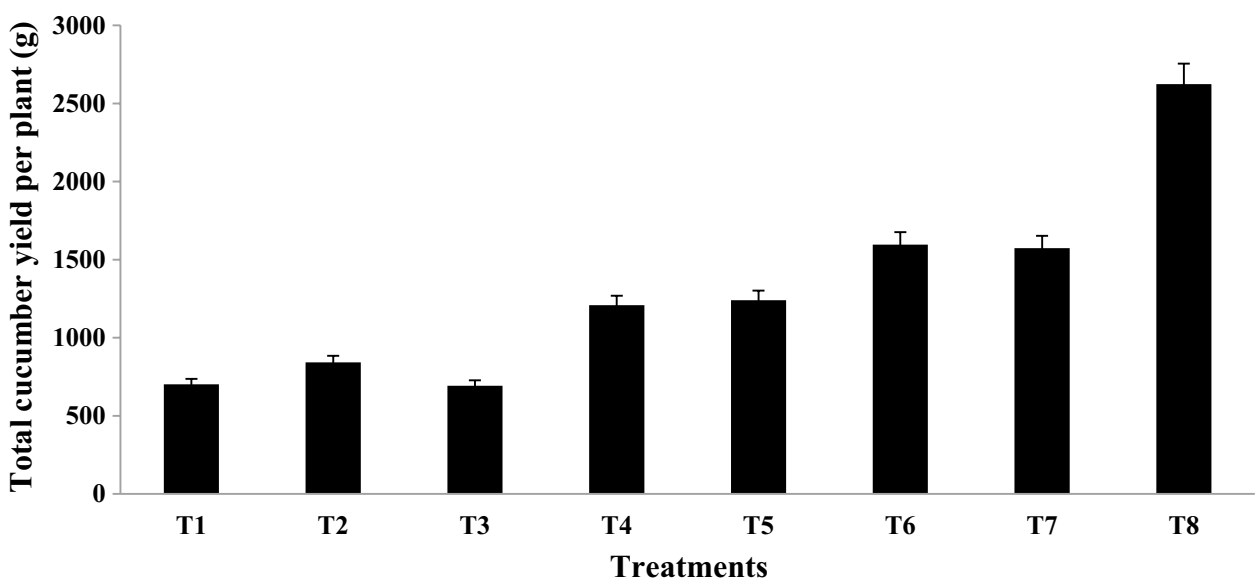

Table 5 Effect of different growing media on NPK contents of cucumber

\begin{tabular}{llll}
\hline Treatments & $\begin{array}{l}\text { Nitrogen contents } \\
(\%)\end{array}$ & $\begin{array}{l}\text { Phosphorus } \\
\text { contents }\left(\mathrm{mg} \mathrm{L}^{-1}\right. \\
\text { DW })\end{array}$ & $\begin{array}{l}\text { Potassium } \\
\text { contents }\left(\mathrm{mg} \mathrm{L}^{-1}\right. \\
\text { DW) }\end{array}$ \\
\hline $\mathrm{T} 1$ & $1.19 \pm 0.20 \mathrm{c}$ & $10.57 \pm 0.42 \mathrm{~d}$ & $\begin{array}{c}80.32 \pm 4.84 \mathrm{~d} \\
\mathrm{~T} 2\end{array}$ \\
$1.37 \pm 0.13 \mathrm{bc}$ & $11.58 \pm 0.23 \mathrm{~cd}$ & $106.98 \pm 4.30 \mathrm{bc}$ \\
$\mathrm{T} 3$ & $1.47 \pm 0.17 \mathrm{abc}$ & $11.09 \pm 0.56 \mathrm{~d}$ & $108.64 \pm 3.97 \mathrm{bc}$ \\
$\mathrm{T} 4$ & $1.85 \pm 0.34 \mathrm{abc}$ & $15.10 \pm 1.39 \mathrm{~b}$ & $117.80 \pm 5.99 \mathrm{~b}$ \\
$\mathrm{~T} 5$ & $1.74 \pm 0.28 \mathrm{abc}$ & $13.61 \pm 0.28 \mathrm{bc}$ & $104.46 \pm 1.60 \mathrm{c}$ \\
$\mathrm{T} 6$ & $1.63 \pm 0.14 \mathrm{abc}$ & $11.12 \pm 0.59 \mathrm{~d}$ & $113.62 \pm 4.30 \mathrm{bc}$ \\
$\mathrm{T} 7$ & $2.02 \pm 0.26 \mathrm{ab}$ & $14.13 \pm 1.16 \mathrm{~b}$ & $140.28 \pm 4.30 \mathrm{a}$ \\
$\mathrm{T} 8$ & $2.16 \pm 0.32 \mathrm{a}$ & $17.14 \pm 1.18 \mathrm{a}$ & $151.94 \pm 3.19 \mathrm{a}$ \\
\hline
\end{tabular}

$\mathrm{T}_{1}=$ silt (control); $\mathrm{T}_{2}=$ compost + silt $(1: 1) ; \mathrm{T}_{3}=$ leaf compost + silt $(1: 1) ; \mathrm{T}_{4}=$ coconut peat + silt $(1: 1) ; \mathrm{T}_{5}=$ perlite + silt $(1: 1) ; \mathrm{T}_{6}=$ compost + leaf compost + silt $(1: 1: 1) ; \mathrm{T}_{7}=$ coconut peat + perlite + silt; $(1: 1: 1)$ and $\mathrm{T}_{8}=$ coconut peat + compost + leaf compost + perlite $+\operatorname{silt}(1: 1: 1: 1: 1)$

\section{Phosphorous contents $\left(\mathrm{mg} \mathrm{L}^{-1}\right)$}

Different potting media exhibited a significant influence on phosphorous $(\mathrm{P})$ contents of the leaves of cucumber plants (Table 5). These media combinations performed better about $P$ uptake than control treatment (silt) where it showed least $P$ contents in the leaves of cucumber leaves. It was observed that highest $\mathrm{P}$ contents $\left(17.14 \mathrm{mg} \mathrm{L}^{-1}\right)$ were observed in $\mathrm{T}_{8}$ (coconut peat + compost + leaf compost + perlite + silt), compared to various other combinations of media.

\section{Potassium contents $\left(\mathrm{mg} \mathrm{L}^{-1}\right)$}

Potassium (K) uptake had also showed marked variations in response to various potting media application for cucumber plants growing (Table 5). The highest $\mathrm{K}$ $\left(151.94 \mathrm{mg}^{-\mathrm{L}}\right.$ ) of cucumber leaves was recorded in $\mathrm{T}_{8}$ (coconut peat + compost + leaf compost + perlite + silt $)$, than other media combinations.

\section{Discussion}

An investigation was carried out to monitor the growth of cucumber plants in pot culture system in response to different growing media. In our present research, various growing media positively influenced the cucumber growth and productivity. It was noted that vegetative and physiological parameters were significantly differed under all media combinations, than control where only silt was used as a growing medium. T8, the combination of coconut peat + compost + leaf compost + perlite + silt and coconut peat + perlite + silt, was found to be most appropriate growing mixtures cucumber plants. Potting media are usually used for a quick emergence of seed, good seedling growth, bedding plants, and nursery raising (Baiyeri 2003). Findings of the present study revealed that organic growing media leaf manure + leaf compost + compost and inorganic perlite + silt decreased the mean emergence time and led to quick growth, as compared to the other treatment. It has been reported that the early emergence depends on media aeration, temperature, and its water content (Guerin et al. 2001). The same results were reported by Shah et al. (2006) as they found that silt and leaf compost in 1:1 proportion, which showed maximum sprouting percentage. The findings are also in accordance with the results of Riaz et al. (2008) who noted the early seedling emergence in zinnia in combination of silt, leaf manure, and compost, and Muriuki et al. (2015) reported the same results in cabbage. It is recommended to use balanced media for cucumber kitchen gardening to attain the suitable plant shoot length. All the media proportions led to beneficial impact for the cucumber growth. The maximum stimulatory impact and the highest improvement in plant height were observed in pots, mixed with perlite, silt, and leaf manure in $(1: 1: 1)$ proportion. This proportion 
gives optimum growth, because $\mathrm{pH}$ and quantity of nitrogen were optimal as reported earlier in lilium by Grassoti et al. (2003). Similar trend was observed by Treder (2008). The leaf chlorophyll contents are also vital for the plant growth as these are directly involved in the photosynthesis (Younis et al. 2015). The number of leaves was found maximum in $T_{8}$ depending upon the combination of media. Similar findings were reported earlier by Riaz et al. (2008) who reported that the highest numbers of leaves were found in combinational treatment, as compared to single growing medium. Potting media have a great impact on root growth, because roots have direct interaction with growing media and such variation in rhizosphere affect the plant growth; hence, its ratio must be in such proportion which provides a balanced pore spaces and solid particles (Riaz et al. 2014). In our present work, fresh and dry biomass differed depending upon the media treatments. Our findings are in accordance with the results of Awang et al. (2010) and Ahmed et al. (2014) as they attained highest fresh and dry weight of Celosia cristata or Carrot (Daucus carota L.) respectively, in combination with more than one growing media. The increase in fresh and dry biomass was also reported by Eklind et al. (2001) under silt and leaf manure combination. The treatment $\mathrm{T}_{8}$ led to maximum accumulation of fresh and dry biomass due to a better moisture retention, aeration, and nutrients. In the current experiment, although leaf chlorophyll contents were positively enhanced in response to various growing media proportions, as compared to control, but plants grown under $\mathrm{T}_{8}$ combination with equal proportion of all media contents exhibited maximum leaf chlorophyll contents. The increase in chlorophyll contents was possibly due to assimilation of maximum nitrogen contents from the growing media to plants. Leaf proline content of the cucumber plants was significantly enhanced. The increased proline contents were due to activation of enzymes of proline biosynthesis as well as a decline in proline oxidation to glutamate, reduced utilization of proline in protein synthesis, and increased the protein turnover eventually leading to higher proline accumulation (Delauney and Verma 1993; Hare and Cress, 1997). Nitrogen has very important impact on the green pigments as it enhances the photochemical reaction rate (Kumar et al. 1988). The similar results were reported by Mahgoub et al. (2006) and Ahmad et al. (2017) as they stated that nitrogen is responsible for the increase of chlorophyll contents in iris leaves and in Coriander leaves, respectively. The increased chlorophyll contents allow the plants to accumulate more photosynthates, subsequently, leading to higher plant growth (Riaz et al. 2014). In the present work, photosynthetic rate of cucumber plants was increased in response to different media combinations. This kind of response is suitable to increase overall growth of plants due to increased photosynthesis and respiration rates (Xu et al. 1995; Lopez et al. 1996). Nitrogen is a major mineral which is essential for normal plant growth and productivity. The highest nitrogen uptake through roots improves translocation of nutrients to leaves (Zhao et al. 2005; Naseem et al. 2015) that ultimately triggers net plant growth (Yasmeen et al. 2012). Similar findings were reported by Mathowa et al. (2014). It has been reported that coconut compost increases nitrogen availability to plants ultimately leading to enhanced growth (Abad et al. 2002). Phosphorus also plays a vital role in nucleic acid biosynthesis, energy metabolism, gas exchange attributes, nitrogen fixation, and enzyme activation (Raghothama 1999). Similarly, potassium is most important mineral nutrient which is responsible for the physiological activities and metabolic processes such as regulation of water in plants, protein biosynthesis, as well as drought tolerance and activation of certain enzymes (Cerda et al. 1995). The availability of increased quantity of organic matter in the growing media enhanced the plant growth due to increased potassium contents in the leaves of plants (Khayyat et al. 2007; Gruda 2009). Therefore, in the current work, overall growth and fruit plant ${ }^{-1}$ of cucumber were enhanced due to improved availability of nitrogen, phosphorous, and potassium nutrients.

\section{Conclusion}

In conclusion, production of cucumber in different growing media showed significant variations in growth and productivity. Combination of coconut peat, compost, leaf compost, perlite, and silt in equal proportion substantially improved the vegetative traits, gas exchange attributes, fruits plant ${ }^{-1}$, and balanced ionic status of the plants. Therefore, the said media combination could be considered suitable for kitchen gardening of cucumber.

Open Access This article is distributed under the terms of the Creative Commons Attribution 4.0 International License (http://creativeco mmons.org/licenses/by/4.0/), which permits unrestricted use, distribution, and reproduction in any medium, provided you give appropriate credit to the original author(s) and the source, provide a link to the Creative Commons license, and indicate if changes were made.

\section{References}

Abad M, Noguera P, Puchades R, Maquieira A, Noguera V (2002) Physico-chemical and chemical properties of some coconut anut dusts for use as peat substitute for containerized ornamental plants. Bioresour Technol 8:241-245. https://doi.org/10.1016/ s0960-8524(01)00189-4

Ahmad T, Shah ST, Ullah F, Ghafoor F, Anwar U (2017) Effect of organic fertilizer on growth and yield of coriander. Int J Agric Environ Res 3(1):116-120

Ahmed A, Sambo E, Arunah B, Uthman Odion EC (2014) Response of farmyard manure and inorganic fertilizers for sustainable yield 
of carrot (Daucus carota L.) in northern Nigeria. J Agric Vet Sci 7:18-25. https://doi.org/10.9790/2380-07221825

Alidoust M, Torkashvand M, Khomami M (2012) The effect of growth medium of peanut shells compost and nutrient solution on the growth of dracaena. Ann Biol Res 3:789-794

Arancon NQ, Edwards CA, Atiyeh R, Metzger JD (2004) Effects of vermicomposts produced from food waste on the growth and yields of greenhouse peppers. Bioresour Technol 93:139-144. https://doi.org/10.1016/j.biortech.2003.10.015

Association of Official Seed Analysis (AOSA) (1990) Rules for testing seeds. J. Seed Technol 12: 1-112. http://www.aosaseed.com/ aosa_rules_for_testing_seeds

Awang Y, Shaharom AS, Mohamad RB, Selamat A (2010) Growth dynamics of Celosia cristata grown in coconut peat, burnt rice hull and kenaf core fiber mixtures. Am J Agric Biol Sci 5:70-76. https://doi.org/10.3844/ajabssp.2010.70.76

Baiyeri KP (2003) Evaluation of nursery media for seedling emergence and early seedling growth of two tropical tree species. Moor J Agric Res 4:60-65. https://doi.org/10.4314/mjar.v4i1.31754

Bates LS, Waldron RP, Teaxe IW (1972) Rapid determination of free proline for water stress studies. Plant Soil 39:205-207

Bremner JM, Mulvaney CS (1982) Nitrogen-Total. In: Page AL, Miller RH, Keeney DR (eds) Methods of soil analysis, Part 2, 2nd edn. Agronomy monograph 9. ASA and SSSA, Madison, WI, USA, pp 595-624

Bulluck LR, Brosius M, Evanylo GK, Ristaino JB (2002) Organic and synthetic fertility amendments influence soil microbial, physical and chemical properties on organic and conventional farms. Appl Soil Ecol 19(2):147-160. https://doi.org/10.1016/S0929 -1393(01)00187-1

Cantliffe DJ, Funes J, Jovicich E, Paranje A, Rodriguez J, Shaw N (2003) Media and containers for greenhouse soilless grown cucumber, melons, peppers and strawberries. Acta Horticulturae 614:199-203

Cerda A, Pardines J, Botella MA, Martinez V (1995) Effect of potassium on growth, water relations, and the inorganic and organic solute contents for two maize cultivars grown under saline conditions. J Plant Nutr 18:839-851. https://doi.org/10.1080/01904 169509364942

Chapman HD, Parker F (1961) Determination of NPK method of analysis for soil, plant and water put. Agricultural University, California

Delauney AJ, Verma DPS (1993) Proline biosynthesis and osmoregulation in plants. Plant J 4:215-223. https://doi.org/10.1046/j.1365313X.1993.04020215

Dorais M, Papadopoulos AP, Gosselin A (2001) Greenhouse tomato fruit quality. Horticul Rev 26:239-319

Eklind Y, Raemert B, Wivstad M (2001) Evaluation of growing media containing farmyard manure compost, household waste compost or chicken manure for the propagation of lettuce (Lactuca sativa L.) transplants. Biol Agric Hortic 19:157-181. https://doi. org/10.1080/01448765.2001.9754919

Ellis RA, Roberts EH (1981) The quantification of ageing and survival in orthodox seeds. Seed Sci Technol 9:373-409 (8182678)

FAO (2011) Food, Agriculture and cities-challenges of food and nutrition security, agriculture and ecosystem management in an urbanizing world. https://www.fao.org/fileadmin/templates/FCIT/ FoodAgriCities_Oct2011

Ghazvini R, Payvast GF, Azarian H (2007) Effect of clinoptiloliticzeolite and perlite mixtures on the yield and quality of strawberry in soil-less culture. Int J Agric Biol 9:885-888 (1560-8530/2007/09-6-885-888)

Gopalan C, Rama SBV, Balasubramanian SC (1989) Nutritive value of Indian foods. National Institute of Nutrition, Indian Council of Medical Research. http://trove.nla.gov.au/work/17516887
Gruda N (2009) Do soilless culture systems have an influence on product quality of vegetables. J Appl Bot Food Qual 82:141-147. doi:https://doi.org/10.18452/9433

Grassoti A, Nesi B, Maletta M, Magnani G (2003) Effects of growing media and planting time on lily hybrids in soilless culture. Acta Hortic 609:395-399. https://doi.org/10.17660/ActaHortic .2003 .609 .60

Guerin V, Lemairea F, Marfa O, Caceres R, Giuffrida F (2001) Growth of Viburnum tinus in peat-based and peat-substitute growing media. Sci Hortic 89:129-142. https://doi.org/10.1016/S0304 $-4238(00) 00228-4$

Gul A, Ergoul D, Ongun R (2005) Comparison of the use of zeolite and perlite as substrate for crisp-head lettuce. Sci Hortic 106:464-471

Hare PD, Cress WA (1997) Metabolic implications of stress induced proline accumulation in plats. Plant Growth Regul 21:79-102. https://doi.org/10.1023/a:1005703923347

Hashemimajd K, Kalbasi M, Goichin A, Shariatmadari H (2004) Comparison of vermicompost and composts as potting media for growth of tomatoes. J Plant Nutr 27:1107-1123. https://doi. org/10.1081/PLN-120037538

Jackson ML (1962) Soil chemical analysis. Constable Co. Ltd, London

Khayyat M, Nazari F, Salehi H (2007) Effects of different pot mixtures on pothos (Epipremnum aureum $\mathrm{L}$ ) growth and development. Am Eur J Agric Environ Sci 2:341-348 (10.1.1.550.7949)

Kumar K, Arvind K, Vidyasagar R, Rao K (1988) Studies on growth and activity of photosynthetic enzymes on Sorghum bicolor L. As influenced by micronutrients. Biol Sci 54:75-79 (54B_1988_1_Art13)

Landauer, K, Brazil M (1985) Tropical home gardens. Selected papers form an international workshop at the Institute of Ecology, Padjadjaran University, Indonesia, December 1985, United Nations University Press

Larcher F, Scariot V (2009) Assessment of partial peat substitutes for the production of Camellia japonica. Hort Technol 2(44):312-316 (hortsci.ashspublications.org/content/44/2/312)

Lopez J, Tremblay N, Voogt W, Dube S, Gosselin A (1996) Effects of varying sulphate concentrations on growth, physiology and yield of the greenhouse tomato. Sci Hortic 67:207-217

Mahgoub HM, Rawia A, Eid A, Bedour H, Abou L (2006) Response of Iris bulbs growth in sandy soil to nitrogen and potassium fertilization. J Appl Sci Res 2:899-903

Maloupa E, Mitsios I, Martinez PF, Bladenopoulou S (1992) Study of substrate use in Gerbera soilless culture grown in plastic greenhouses. Acta Horticul 323:139-144. http://www.actahort.org/ members/showpdf?booknrarnr=323_12

Mathowa T, Bosenakitso M, Mojeremane W, Mpofu C, Legwaila GM (2014) Effect of growing media on seedling growth of African baobab (Adansonia digitata L.). Int J Adv Res Biol Sci 1:94-104. http://www.ijarbs.com/pdfcopy/oct2014/ijarbs14

Moya JL, Gomez-Cademas A, Primo-Millo E, Talon M (2003) Chloride absorption in salt-sensitive Carrizo citrange and salt tolerant Cleapatra mandarian citrus rootstocks is linked to water use. $\mathrm{J}$ Exp Bot 54:825-833

Muriuki AW, Schnier HF, Recke H, Kanyanjua SM (2015) The effect of farmyard manure and fertilisers on cabbage yields in Kenya. East Afr Agric For J 68(1):41-49. https://doi.org/10.4314/eaafj .v68i1.1775

Nair A, Ngouajio M, Biernbaum J (2011) Alfalfa-based organic amendment in peat-compost growing medium for organic tomato transplant production. HortScience 46:253-259. http://hortsci.ashsp ublications.org/content/46/2/253

Naseem MK, Khan MA, Younis A, Ahmad R (2015) Exploiting the nutritional requirement for growth, flower production and phytochemical profile of Murraya exotica. Pak J Agric Sci 52:379-386. https://doi.org/10.12692/ijb/10.4.151-159 
Nkosi S, Gumbo T, Kroll F, Rudolph M (2014) Community gardens as a form of urban household food and income supplements in African cities: experiences in Hammanskraal, vol 112. Africa Institute of South Africa Briefing, p 1-6. https://doi.org/10.1080/19463 138.2017.1286349

Noreen Z, Ashraf M (2009) Assessment of variation in antioxidative defense system in salt- treated pea (Pisum sativum) cultivars and its putative use as salinity tolerance markers. J Plant Physiol 166:1764-1774. https://doi.org/10.1016/j.jplph.2009.05.005

Olsen SAR, Sant MD, Gislerod HR, Solbraa K (1984) Nitrogen balance in bark composts used as growing media. Acta Hortic 150:193-202

Olympios CM (1999) Overview of soilless culture: advantages, constraints and perspectives for its use in Mediterranean countries. Options Mediterr 31: 307-324. http://soil-science.iauahvaz.ac.ir/ Files/Forms/2011-10-24_06.15.52_6

Raghothama KG (1999) Phosphate acquisition. Ann Rev Plant Physiol Plant Mol Biol 50:665-693. https://doi.org/10.1146/annurev.arpla nt.50.1.665

Riaz A, Arshad M, Younis A, Raza A, Hameed M (2008) Effect of different growing media on the growth and flowering of Zinnia elegans cv. Blue point. Pak J Bot 40:1579-1585

Riaz A, Farooq U, Younis A, Karim A, Taj AR (2014) Growth responses of Zinnia to different organic media. Acta Hort 1018:565-572. https://actahort.org/books/1018/1018_62.htm

Sarwar M, Rehman S, Ayyub CM, Ahamd W, Shafi J, Shafique K (2013) Modeling growth of cut flower stock (Matthiola incana) in response to differing in nutrient level. Univers J Food Nutria Sci 1(1):4-10. https://doi.org/10.13189/ujfns.2013.010102

Sarwar M, Amjad M, Ayyub CM (2017) Alleviation of salt stress in cucumber (Cucumis sativus L.) through seed priming with triacontanol. Int J Agric Biol 19:771-778. https://doi.org/10.17957 /IJAB/15.0356

Savvas D, Samantouros K, Paralemos D, Vlachakos G, Stamatakis M, Vassilatos C (2004) Yield and nutrient status in the root environment of tomatoes grown on chemically active and inactive inorganic substrates. Acta Horticul 644:377-383 (644/644_50.htm)

Shah M, Khattak AM, Amin N (2006) Effects of different growing media on the rooting of Ficus binnendijkii 'Amstel Queen' cuttings. J Agric Biol Sci 1(3):15-17

Steel RGD, Torrie JH, Dicky DA (1997) Principles and procedures of statistics: a biometric approach. Mc Graw Hill Inc, New York

Stepien P, Klobus G (2006) Water relations and photosynthesis in Cucumis sativus L. leaves under salt stress. Biol Plant 50:610616. https://doi.org/10.1007/s10535-006-0096-z

Talukder A, Kiess L, Huq N, De-pee S, Darton-Hill Bloem MW (2002a) Increasing the production and consumption of vitamin A-rich fruits and vegetables: lesson learned in taking the Bangladesh homestead gardening programme to national scale. Food Nutr Bull 21(2):165-172

Talukder A, Kiess L, Huq N, De-pee S, Darton-Hill Bloem MW (2002b) Increasing the production and consumption of vitamin A-rich fruits and vegetables: lesson learned in taking the Bangladesh homestead gardening programme to national scale. Food Nutr Bull 21(2):165-172

Tariq U, Rehman S, Khan MA, Younis A, Yaseen M, Ahsan M (2012) Agricultural and municipal waste as potting media components for the growth and flowering of Dahlia hortensis 'Figaro'. Turk J Bot 36:378-385. https://doi.org/10.3906/bot-1109-16

Thomas GW (1996) Soil pH and soil acidity. In: Sparks DL, Page AL, Helmke PA, Loeppert RH, Soltanpour PN, Tabatabai MA, Johnton CT, Sumer ME (eds) Method of soil analysis. Part 3.
Chemical Methods. Book Series No. 5 SSSA, Madison Wisconsin, pp 475-490

Treder J (2008) The effects of coconut peat and fertilization on the growth and flowering of oriental lily 'star gazer'. J Fruit Ornam Plant Res 16:361-370 (agro-article-7afd2b13-45a9-4494-aa53-8ef8e16aeab7)

U.S. Salinity Lab. Staff (1954) Diagnosis and Improvement of Saline and Alkali Soils. Agricultural Handbook No. 60. United State Department of Agriculture

Vaughn SF, Deppe NA, Palmquist DE, Berhow MA (2011) Extracted sweet corn tassels as a renewable alternative to peat in greenhouse substrates. Ind Crops Prod 33:514-517

Wang SY, Lin SS (2006) Composts as soil supplement enhanced plant growth and fruit quality of strawberry. J Plant Nutr 25:2243-2259. https://doi.org/10.1081/PLN-120014073

Watanabe FS, Olsen SR (1965) Test of an ascorbic acid method for determining phosphorus in water and $\mathrm{NaHCO}_{3}$ extracts from soil. Soil Sci Soc Am Proc 29:677-678. https://doi.org/10.2136/sssaj 1965.03615995002900060025x

Wilson SB, Stoffella PJ, Graetz DA (2003) Compost amended media and irrigation system influence containerized perennial Salvia. J Am Soc Hortic Sci 128:260-268. http://journal.ashspublication s.org/content/128/2/260.short

Xu HL, Gauthier L, Gosselin A (1995) Effects of fertigation management on growth and photosynthesis of tomato plants grown in peat, rockwool and NFT. Sci Hortic 63:11-20

Yasmeen S, Younis A, Rayit A, Riaz A, Shabeer S (2012) Effect of different substrates on growth and flowering of Dianthus caryophyllus cv. 'Chauband Mixed'. Am-Eurasian. J Agric Environ Sci 12:249-258. https://www.idosi.org/aejaes/jaes12(2)12/18

Younis A, Riaz A, Khosa SS, Rayit A, Yasmeen S (2011) Effect of foliar application of macro and micro nutrients on growth and flowering of Gerbera jamesonii L. Amer-Eurasian. J Agric Environ Sci 11:736-757. https://www.idosi.org/aejaes/jaes11(5)11/20

Younis A, Riaz A, Sajid M, Mushtaq N, Ahsan M, Hameed M, Tariq U, Nadeem M (2013) Foliar application of macro- and micronutrients on the yield and quality of Rosa hybrid cvs. Cardinal and Whisky Mac. Afric J Biotechnol 12:702-708. https://doi.org/10.5897/ ajb12.2226

Younis A, Riaz A, Javaid F, Ahsan M, Tariq U, Aslam S, Majeed N (2015) Influence of various growing substrates on growth and flowering of potted miniature rose cultivar "Baby Boomer'. Curr Sci Perspect 1:16-21

Zaller JG (2007) Vermicompost as a substitute for peat in potting media: Effects on germination, biomass allocation, yields and fruit quality of three tomato varieties. Sci Horticul 112:191-199. https://doi.org/10.1016/j.scienta.2006.12.023

Zekri M (1991) Effects of $\mathrm{NaCl}$ on growth and physiology of sour orange and Cleopatra mandarin seedlings. Sci Horticul 47:305315. https://doi.org/10.1016/0304-4238(91)90013-O

Zhao DKR, Reddy KR, Kakani VG, Reddy VR (2005) Nitrogen deficiency effects on plant growth leaf photosynthesis, and hyperspectral reflectance properties of sorghum. Euro J Agron 22:391403. http://www.ars.usda.gov/research/publications/publicatio $\mathrm{n} /$ ?seqNo115=166050

Publisher's Note Springer Nature remains neutral with regard to jurisdictional claims in published maps and institutional affiliations. 